\title{
High-resolution deployable CubeSat prototype
}

\author{
Noah Schwartz, William Brzozowski, Maria Milanova, \\ Katherine Morris, Stephen Todd, Zeshan Ali, \\ Jean-Francois Sauvage, Alex Ward, David Lunney \\ and Donald MacLeod
}

\section{Published version information:}

Citation: N Schwartz et al. 'High-resolution deployable CubeSat prototype.' Proceedings of SPIE, vol. 11443 (2020): 1144331. Is in proceedings of: SPIE Astronomical Telescopes + Instrumentation 2020, Online Only, United States, 1418 Dec 2020.

DOI: $\underline{10.1117 / 12.2562255}$

(C)2020 Society of Photo-Optical Instrumentation Engineers (SPIE). One print or electronic copy may be made for personal use only. Systematic reproduction and distribution, duplication of any material in this publication for a fee or for commercial purposes, and modification of the contents of the publication are prohibited.

This version is made available in accordance with publisher policies. Please cite only the published version using the reference above. This is the citation assigned by the publisher at the time of issuing the APV. Please check the publisher's website for any updates. 


\section{High-resolution deployable CubeSat prototype}

Schwartz, Noah, Brzozowski, William, Milanova, Maria, Morris, Katherine, Todd, Stephen, et al.

Noah Schwartz, William Brzozowski, Maria Milanova, Katherine Morris, Stephen Todd, Zeshan Ali, Jean-Francois Sauvage, Alex Ward, David Lunney, Donald MacLeod, "High-resolution deployable CubeSat prototype," Proc. SPIE 11443, Space Telescopes and Instrumentation 2020: Optical, Infrared, and Millimeter Wave, 1144331 (13 December 2020); doi: 10.1117/12.2562255

SPIE. Event: SPIE Astronomical Telescopes + Instrumentation, 2020, Online Only 


\title{
High-resolution deployable CubeSat prototype
}

\author{
Noah Schwartz*a, William Brzozowski ${ }^{\mathrm{a}}$, Maria Milanova ${ }^{\mathrm{a}}$, Katherine Morris ${ }^{\mathrm{a}}$, Stephen Todd ${ }^{\mathrm{a}}$, \\ Zeshan Ali ${ }^{\mathrm{a}}$, Jean-Francois-Sauvage ${ }^{\mathrm{b}, \mathrm{c}}$, Alex Ward ${ }^{\mathrm{d}}$, David Lunney ${ }^{\mathrm{a}}$, Donald MacLeod ${ }^{\mathrm{a}}$ \\ aUK Astronomy Technology Centre, Blackford Hill, Edinburgh EH9 3HJ, United Kingdom; \\ bONERA, 29 avenue de la Division Leclerc, 92322 Châtillon, France; ' ${ }^{\mathrm{c} A i x}$ Marseille Univ, CNRS, \\ CNES, LAM, Marseille, France; ${ }^{\mathrm{d} R a z o r b i l l ~ I n s t r u m e n t s ~ L t d, ~ U n i t ~} 8$ Castlebrae Business Centre, \\ Edinburgh EH16 4BB, United Kingdom.
}

\begin{abstract}
The volume available on-board small satellites limit the optical aperture to a few centimetres, which limits the GroundSampling Distance (GSD) in the visible to approximately $3 \mathrm{~m}$ at $500 \mathrm{~km}$. We present the latest development of a laboratory demonstrator for a deployable telescope that will triple the achievable ground resolution and quadruple the photometric capability from a CubeSat imager.

In this paper, we present the overall opto-mechanical design of a Cassegrain telescope with a segmented primary mirror with a $30 \mathrm{~cm}$ baseline. The segments are folded for launch and unfold in space. To enable diffraction-limited imaging, piston, tip, and tilt (PTT) on each of the mirror segments should be below $12 \mathrm{~nm}$ RMS. The key challenge is to ensure phasing, and this precision level will require an active phasing stage.

We present laboratory results of deployment and active phasing of the primary mirror segments. The initial deployment is performed using shaped memory alloy that deploy mirror segments. We demonstrate a repeatability below $\pm 4.5 \mu \mathrm{m}$, enabling the four PSFs (one for each mirror segment) to be imaged on the detector simultaneously. An alignment step using compact and calibrated capacitive sensors allows for a control of the mirror positions in PTT below the wavelength. Finally, we investigate the sensitivity of misalignments of a deployable secondary mirror and show that it is well within reach of the technology developed in this study.
\end{abstract}

Keywords: Active optics, CubeSat, Co-phasing, Wavefront sensing, Deployable optics

\section{INTRODUCTION}

To reach their full potential, many scientific and technological fields (e.g. Earth climate monitoring and protection, defence and security, and Solar system exploration) require very high-resolution images as often as possible, combining highresolution images and high revisit rates. However, combining both high spatial and temporal resolution is, at the moment, out of reach at reasonable costs. Indeed, both requirements can only be achieved simultaneously by using multiple satellites in a LEO (Low Earth Orbit) constellation, which requires small individual satellites to lower the cost. However, using small platforms (e.g. CubeSat, a miniaturised standard satellite) constrains the size of optical apertures, limiting the spatial resolution. For instance, a $10 \mathrm{~cm}$ diameter telescope (typical maximum aperture on board a CubeSat) provides only $3 \mathrm{~m}$ resolution images from a $500 \mathrm{~km}$ orbit in visible wavelength $(500 \mathrm{~nm})$ due to the diffraction limit. Developing an optical aperture greater than $10 \mathrm{~cm}$ on a CubeSat represents a major opto-mechanical challenge.

This project aims at opening a new parameter space in the trade-off between resolution and revisit time by developing a deployable optical telescope on a CubeSat platform. By a breakthrough in performance over current state of the art this will provide high-performance payloads - comparable to larger more expensive satellites - while maintaining low-costs. It has the potential of becoming a new off-the-shelf standardized system to be used on many high angular resolution and high revisit space missions on board CubeSats or similar nanosats. (Figure 1-1 shows an illustration of a 6U CubeSat, with $1 \mathrm{U}$ being a standard CubeSat volume of $10 \times 10 \times 10 \mathrm{~cm}^{3}$ cubic units.

*noah.schwartz@stfc.ac.uk

Space Telescopes and Instrumentation 2020: Optical, Infrared, and Millimeter Wave, edited by Makenzie Lystrup, Marshall D. Perrin. Proc. of SPIE Vol. 11443, 1144331 · C) 2020 SPIE

CCC code: $0277-786 X / 20 / \$ 21 \cdot$ doi: $10.1117 / 12.2562255$ 


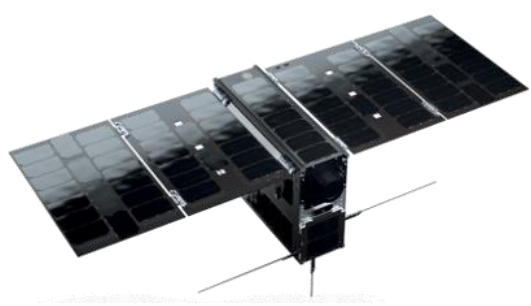

Figure 1-1: 6U EPIC platform by AAC Clyde Space. It offers a 4.5U payload volume, a $120 \mathrm{~W}$ peak payload power, and a pointing stability of 7.2 arcsec. On a larger $12 \mathrm{U}$ platform it is possible to double payload volume and power.

In this paper, we present an innovative mechanical design for packaging (to fit into the CubeSat volume), deploying, and controlling the position of the primary mirror petals. We report on the development of a full end-to-end laboratory experimental setup to validate the deployment and alignment of the primary mirror (M1) segments. In section 2, we present the general concept to achieve a 1 meter ground sampling distance from a CubeSat. In Section 3, we show the overall performance of the mirror segment deployment mechanism using shape memory alloy, and of the fine alignment control using a combination of linear piezo actuator and miniaturised position sensors. Finally, in Section 4 we explore potential secondary mirror (M2) deployment designs and in particular the requirements in terms of alignment precision.

\section{1 METER RESOLUTION FROM STANDALONE DEPLOYABLE TELESCOPE}

\subsection{High-resolution on a CubeSat}

Major science using deployable telescopes will be realised with the James Webb Space Telescope (launch 2021, cost $>\$ 8 \mathrm{Bn})$. However, no affordable deployable optical telescope currently exists. We propose a highly innovative approach to give access to high spatial resolution on small platforms, leveraging low cost CubeSat standards to enable affordable constellations and ensure high revisit rates.

UKATC's deployable CubeSat will offer a 1 meter ground resolution from a LEO with a $30 \mathrm{~cm}+$ optical aperture deployed and aligned in space (diffraction-limit being $0.8 \mathrm{~m}$ at $500 \mathrm{~km}$ for a $500 \mathrm{~nm}$ wavelength). For a fixed ground sampling distance, this will also improve photometric capacities and signal-to-noise ratio, simply by providing a larger collecting surface area. We aim at using a standard $6 \mathrm{U}$ CubeSat of $<10 \mathrm{~kg}$ to deploy a $30 \mathrm{~cm}$ telescope in space [1, 2]. As a comparison, the mass of the SPOT6/7 satellites is $714 \mathrm{~kg}$ in order to achieve $1.5 \mathrm{~m}$ Ground Sampling Distance (GSD). This gain in mass directly translates into a gain in cost and therefore allows multiplying the number of platforms and increasing the temporal coverage by a factor 10 to 20 .



Figure 2-1: Image ground resolution (GSD) versus satellite mass and swath for deployable CubeSat and a selection of major satellites already in orbit. The bubble size represents swath. Orange bubbles represent CubeSats. The dashed red square represents a currently unexploited region in terms of mass and resolution. 
We show Figure 2-1 an illustration of image ground sampling distance (GSD) versus satellite mass and swath (portion of the ground seen by the imager) for a selection of satellites. CubeSats are highlighted in orange, and demonstrates the clear gain in mass brought by the use of small satellites. This graph also clearly indicate the currently unexploited region in terms of mass and ground resolution addressed by this work (under the label "Deployable CubeSat").

By using a constellation of satellites, it is possible to lower the waiting time between observations of a given ground scene. However, vastly increasing the number of satellites in orbit is only attractive if individual satellites can be manufactured at a fraction of the cost. Providing average prices for EO products is difficult, as the acquisition cost of an image depends on a large number of factors. Focusing on the individual satellites themselves, we estimate the rough order of magnitude (ROM) costs of repeat builds of the same satellite design to be 10 to 100 times below current mission costs. We are able to provide this level of cost reduction both by drastic miniaturisation (deployable optics) which reduces launch costs, and standardisation (CubeSats).

\subsection{General concept}

UKATC's prototype provides a first demonstrator for a new payload technology: a standalone and automated deployable telescope dedicated to optical imaging. On a conceptual level (see Figure 2-2), deployable optical telescopes are composed of a primary mirror (M1, principal light-gathering surface), a secondary mirror (M2, folding light towards the detector), a baffle to stop unwanted light, and an active optics control to guarantee final optical quality. Finally, it often requires field correcting optics to compensate for field-dependent aberrations. Active and adaptive control of segmented primary mirrors a key enabling technology for space-based science (e.g. for instruments such as MIRI [4] on the James Webb Space Telescope instrument) and for ground-based astronomy [ $\underline{5}, \underline{6}]$. However, transposing this technology to nanosats represents a major innovation.

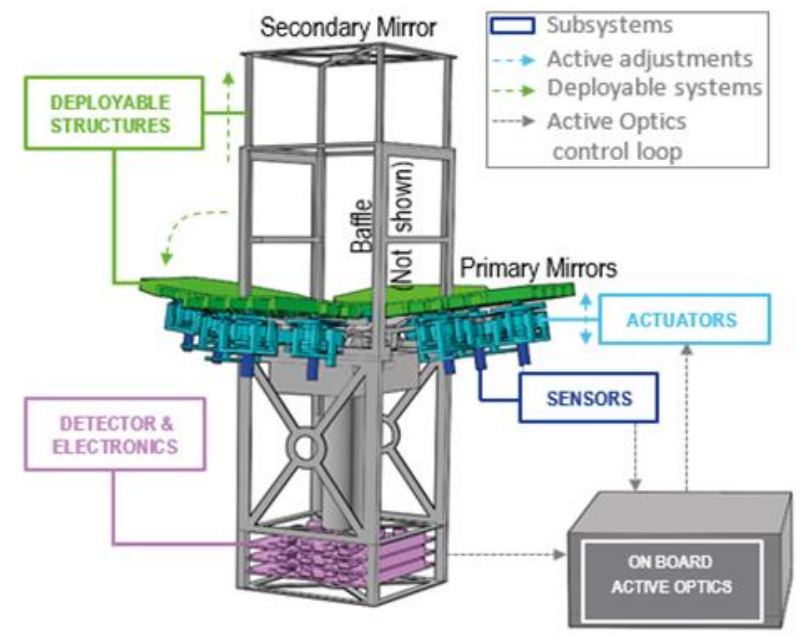

Figure 2-2: Simplified illustration of the payload concept: deployable structures (primary \& secondary mirrors, baffle), actuators to adjust mirror positions, sensors to measure mirror positions, detector to assess image quality, and on-board computer to control and adjust mirror positions to reach diffraction-limited image quality (i.e. active optics).

The mechanical repeatability that deployable structures can achieve is typically of the order of a few microns $[1,3]$. To take full advantage of a large deployable aperture and reach the highest possible resolution, optical surfaces need to be aligned to approx. $10 \mathrm{~nm}$, a factor approx. 500. In addition, in-orbit disturbances may affect the optical stability of the system over short and long timescales. We therefore aim to incorporate an active correction to compensate for static and dynamic aberrations. Actuators (Figure 2-2) provide movement to the optical surfaces. Their extension must be greater than the deployment repeatability (i.e. several microns), and their resolution smaller than the allowable errors (i.e. tens of $\mathrm{nm}$ ) [3]. The control feedback loop is provided by dedicated on board algorithms.

\section{M1 PROTOTYPE DESIGN AND LABORATORY EXPERIMENTATION}

In order to demonstrate the feasibility of packaging and controlling large mirror segments into a small volume, the UKATC has developed a laboratory demonstration. This prototype concentrates on the main challenge: the deployment of the primary mirror M1. For the purpose of the demonstrator, we constrained the payload volume allocated to M1 alone to $1.5 \mathrm{U}$ 
(other elements such as the secondary mirror, baffle, detector, etc. taking up the remaining of the volume). We demonstrate that not only it is possible to stay within the prescribed volume envelope but that the mirror segments can be deployed with micrometre precision, and controlled in position with nanometre precision. We present in this section an innovative mechanical design for packaging (to fit into the CubeSat volume during launch), deploying (while in orbit), and controlling with high accuracy the position of the primary mirror segments.

\subsection{Optical design overview}

The imaging system of the deployable CubeSat is based around a Cassegrain telescope with a lens assembly to set the correct focal length and to provide aberration correction across the field-of-view (FoV). The longest baseline across our segmented primary mirror is $300 \mathrm{~mm}$, giving diffraction limited resolution of the system of 0.38 arcsec at $550 \mathrm{~nm}$. At an altitude of $500 \mathrm{~km}$ this corresponds to $0.92 \mathrm{~m}$ on the ground. Typical CMOS imaging detectors have pixels of around $5.5 \mu \mathrm{m}$ square. A ground sampling distance (GSD) of $1 \mathrm{~m} / \mathrm{pixel}$ implies a focal length of $2750 \mathrm{~mm}$ (focal ratio of f/9.2). A CMOS detector with $2 \mathrm{k}$ x $2 \mathrm{k}$ pixels will therefore give an instantaneous FoV of $2 \mathrm{~km}^{2}$.
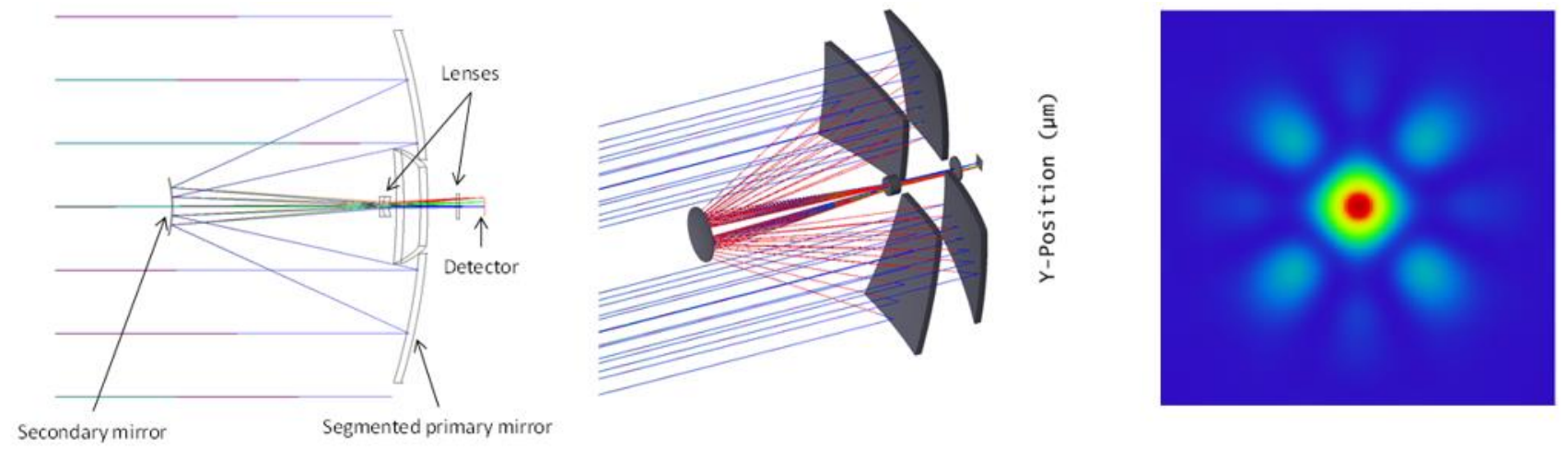

Figure 3-1: (Left) Two different views of the optical design. (Right) The diffraction limited point-spread function.

Analysis shows that the system is extremely sensitive to small misalignments of the mirror segments. This is not surprising when we consider that we are trying to form a single optical surface of diffraction limited performance. There is a very high sensitivity to decenter of the segments - even a 1 micron decenter has a significant effect. This is a result of the very fast focal ratio of the primary mirror (f/0.75). This misalignment can be compensated to some degree by adjusting the tilt and focus of the segment. This sensitivity to decenter can be reduced by increasing the radius of curvature of the primary mirror. This would require an increase in the separation between M1 and M2, making the telescope longer (see section 4). This would require a more complex deployment mechanism for M2, but does have a dramatic effect on the sensitivity, with tolerances relaxed by a factor of $\sim 6.5$ if the M1-M2 distance is increases from $200 \mathrm{~mm}$ to $400 \mathrm{~mm}$. Such a change would do nothing to relax the resolution requirements for the tilt and focus adjustments of the segments. These sensitivities are independent of the optical design of the telescope, and simply reflect the deviation of the surface of the segment relative to the nominal optical surface.

\subsection{Mechanical design overview}

Figure 3-2 shows an overview of the prototype CubeSat system and its major sub-components. A modular integration of the CubeSat systems presented below was developed for the prototype laboratory demonstrator. This allowed easy access to all the various components for inspection, alignment and modification during the test programme. All structures are predominantly aluminium (including the mirrors) to ensure any differential thermal contraction effects are minimised. Special features and shims for component alignment and adjustment were incorporated into the mirrors and sensors. Much of the integration work was the adjustment of machinable shims to set the baseline mirror positions. The secondary mirror (M2) was supported statically above the CubeSat main structure on an optical bench. The deployment and automatic adjustment of the M2 mirror was beyond the scope of the current work; however an analysis is presented section 4 . 


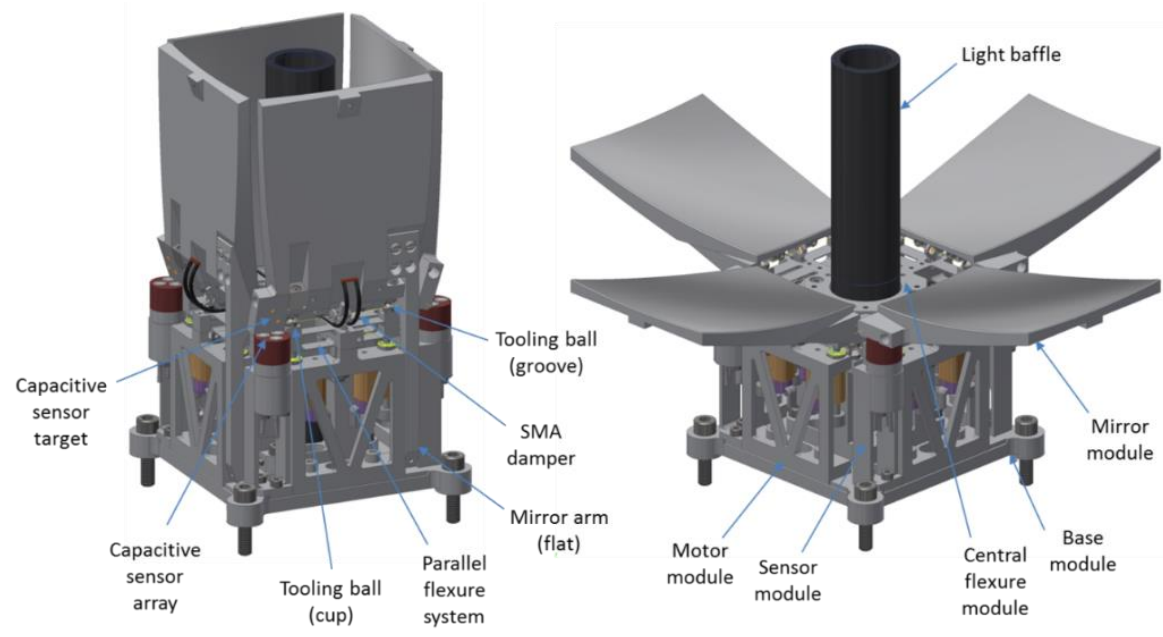

Figure 3-2: Overview of the mechanical design of the prototype CubeSat. Left: folded form for launch, fitting into a 1.5U volume. Right: deployed state reached after launch, deploying a $300 \mathrm{~mm}$ optical aperture.

The deployment motion is provided by an extension spring. It is mounted on a short lever arm that is designed to produce zero torque on the deployment axis when the mirror is in its final position. This is important so that the spring does not interfere with the tip-tilt adjustments on the mirror. Spring deployment mechanisms can cause undesirable shock or vibration issues when released. These undamped motions can cause repeatability issues in precision mechanisms. To prevent this from happening the mirror motion is controlled by the use of a Shape Memory Alloy (SMA), in this case Nitinol wire [7].

Three motors enable each mirror to be further manipulated in tip, tilt and piston. The motor chosen for this application is the Newport 8354 Tiny Picomotor. They have a $30 \mathrm{~nm}$ positioning resolution, a high actuation force $(13 \mathrm{~N})$, and have the ability to hold position while powered off

\subsection{Experimental setup and testing}

The purpose of the demonstrator was to test, in an experimental setup, the deployment and fine position control of the primary mirror segments. The 4-segment primary mirror design is presented section 3.2 and uses a fixed secondary mirror. The light source assembly is composed of a reflective LCD (QXGA-R9 from Forth Dimension Displays) to project a 2048x1536 pixels extended scene. It also contain a single mode fibre for diffraction limited illumination. We use a commercial Orion VX12L Newtonian telescope to project a $300 \mathrm{~mm}$ collimated beam onto the deployable CubeSat demonstrator. Newtonian telescopes have a central obstruction due to the presence of the secondary mirror in the light path, however we can accept a central obscuration of up to $100 \mathrm{~mm}$ diameter to match our segmentation pattern. Finally, a Flea3 1.3 MP Mono USB3 Vision Point Grey camera provides a 1280×1024 image.

Figure 3-3 shows the experimental setup used for laboratory testing. The presented vertical setup was later changed to a horizontal setup and baffles added to minimise vibrations, unwanted light, and airflow. This figure shows the main subsystems, including: the light source assembly, the Newtonian telescope providing a $300 \mathrm{~mm}$ collimated illumination, the fixed secondary mirror assembly, the camera assembly, and the deployable primary mirror assembly. It presents a CAD model of the system, the prototype as tested in the lab, and a typical extended scene image obtain with the setup. 

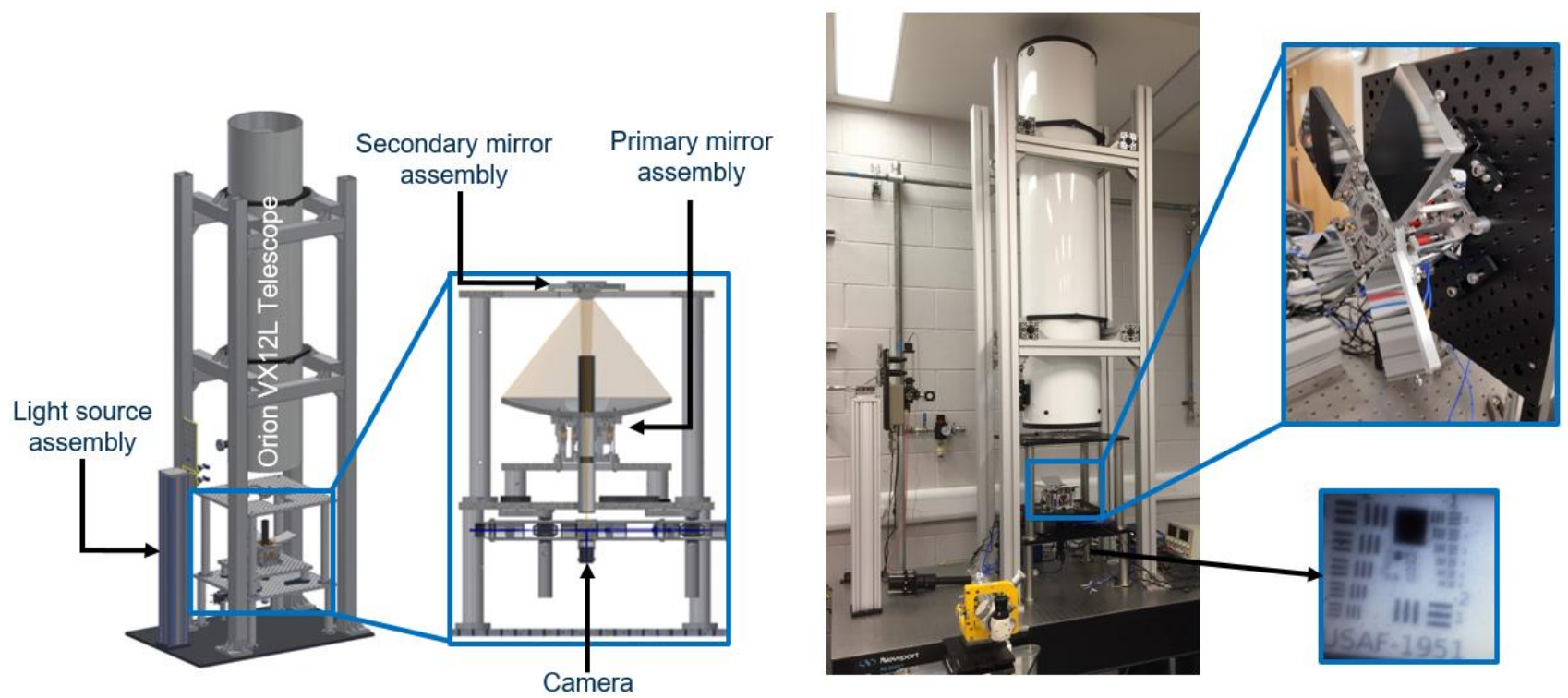

Figure 3-3: Experimental setup showing the commercial Newtonian telescope to provide $300 \mathrm{~mm}$ collimated illumination, the light source assembly to project an extended objects or a diffraction limited source. Left: CAD model of the system. Right: experimental setup in the lab, showing the M1 assemble and a typical extended scene image. Vertical setup was changed to horizontal and baffled to minimise vibrations, unwanted light, and airflow.

\subsection{Prototype experimental results: deployment accuracy}

In order to produce a high optical quality, it is required that the mirrors are deployed with adequate repeatability and precision. To test the deployment performance, the mirrors were retracted and deployed into their kinematic mounts 500 times. Each time the mirror settled into its mount a reading from three commercial off-the-self high-precision MicroEpsilon CapaNCDT CSE05 capacitive sensors was taken. Figure 3-4 shows the distribution of the measurement data for each sensor. The red line shows a normal distribution fit to the data with mean subtracted. From the figures it can be seen that in all instances the $2 \sigma$ variation ( $95 \%$ confidence) around the mean is well within our required repeatability tolerance of $\pm 10 \mu \mathrm{m}$. Sensors 1 and 2 show a close approximation to a normal distribution, but sensor 3 has a bias toward the left tail. The reason for this bias is not clear and more testing is required to determine its source.
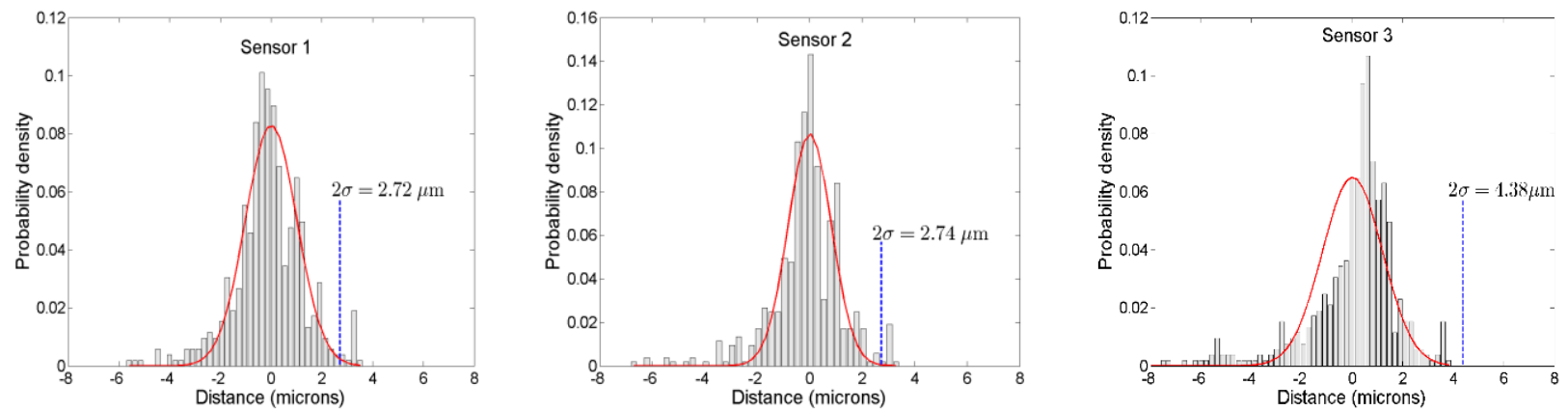

Figure 3-4: Deployment repeatability histograms for the three sensors. A best fit normal distribution for each chart is shown in red.

\subsection{Embedded position sensors}

After the initial deployment from a folded to an unfolded state (see Figure 3-2), we use calibrated position sensors to align the mirrors more accurately. In collaboration with Razorbill Instrumentation, we have embedded the ZED-CAP [ 8 ] - a non-contact displacement sensor - into our prototype. It delivers high measurement precision but has a low power consumption and small volume. It performs a fine alignment of two surfaces with a precision up to $2 \mathrm{~nm}$, and has a total volume $<1 \mathrm{~cm}^{3}$ (with no additional electronics required, providing an $\mathrm{I}^{2} \mathrm{C}$ output directly from the sensor), 
Like many capacitive sensors, the ZED-CAP uses a change in capacitance between two capacitor plates to determine a displacement. As the two sense heads approach each other, the signal increases and the resolution goes up, leading to an initial 'coarse mode' with a resolution of $20 \mathrm{~nm}$ followed by a 'fine mode' with a resolution of $2 \mathrm{~nm}$. This is ideal for optical alignment and phasing in which a long range may be required, but high resolution is only a necessity very near the target displacement. Figure 3-5 show an image of the sensors integrated into UKATC's experimental setup.



Figure 3-5: Razorbill Instrument's gold-plated ZED-CAP sensors in use on the UKATC experimental prototype. Three sensors are in use to control each mirror segment to provide feedback on the three degrees of freedom: tip, tilt and piston.

A major advantage of the ZED-CAP is that it is optimized to give the highest possible resolution when measuring small gaps. The measurement noise (which is the limiting factor in the sensor performance) gets smaller and smaller as the two parts of the sensor are brought together (see Figure 3-6 left). This means that the sensor increases in sensitivity as the optical surface approaches the target position allowing the greatest precision where it is most needed. As an illustration of ZED-CAP resolution, graph Figure 3-6 (right) shows the sensor output for $2 \mathrm{~nm}$ steps in a dedicated piezoelectric test fixture. The $2 \mathrm{~nm}$ steps are clearly detectable, and some light post-processing can greatly increase measurement accuracy.


Figure 3-6: ZED-CAP performance. (Left) Accuracy of measurement as a function of the distance between the 2 parts.

(Right) Illustration of noise levels for a $2 \mathrm{~nm}$ step.

\subsection{Prototype experimental results: alignment using close-loop control}

We use the ZED-CAP capacitive sensor and the PicoMotor actuators in conjunction to drive the mirrors into a desired position. We use a classical integrator control: $u_{n+1}=u_{n}-g M_{\text {control }} s_{k}$, where $M_{\text {control }}$ is the control matrix, $g$ is the uniform scalar gain of the integrator and is set to $0.5, u_{n}$ is the PicoMotors control vector at iteration step $n$, and $s_{k}$ is the capacitive sensor measurements. $\mathbf{M}_{\text {control }}$ is calculated from the generalised pseudo-inverse of the interaction matrix which itself is measured by recording the response on the position sensors of each motor sequentially. Figure 3-7 shows the 3 capacitive channel measurements taken from one of the four mirrors. The initial error to the reference is $4.4 \mu \mathrm{m}, 3.7 \mu \mathrm{m}$, and $2.6 \mu \mathrm{m}$ for the 3 channels respectively. 



Figure 3-7: (Left) error as measured from the 3 displacement sensor channels as a function of iteration step. (Right) Example of the 4 individual PSFs before alignment; with inset showing a zoom of PSF aligned but not yet co-phased.

After an initial very fast convergence (only 10 iterations to bring the error down from several microns to approx. <16 nm), the active optics loop appears to struggle to bring the error down below this level with noticeable oscillations. This has been identified to come mainly from the actuators used to drive the mirrors into position, and not the sensors themselves. In spite of the limited motor step size of approx. $30 \mathrm{~nm}$, hysteresis and backlash (in addition to some limited amount of stiction in the system itself), we are able to converge to a final error $<5 \mathrm{~nm}$. This is below our requirement of $12 \mathrm{~nm}$ per mirror segment degree of freedom to reach diffraction-limited performance. It is important at this point to note that an initial calibration step is required to measure the absolute datum (i.e. reference mirror position for which perfect alignment, or co-phasing, is achieved). These positions are then used in the close loop operation as the reference.

\subsection{Active optics control}

The reference positions for each mirror required to reach diffraction-limited performance (see section 3.6) can be measured and set during integration and tests of the telescope on the ground. However, in operation the payload will likely be subject to vibrations during launch and the space environment including vibrations, radiation and transient thermal loading. In order to maintain alignment and diffraction-limited performance, on-board active control of the mirrors is required. Because of the limited CubeSat volume and power, it is not possible to add a dedicated wavefront sensor and only focal plane images can be used. We present is this section control of the mirrors' position using focal plane sharpening, which has the advantage of only requiring the focal plane image and can operate both a point source and an extended source.
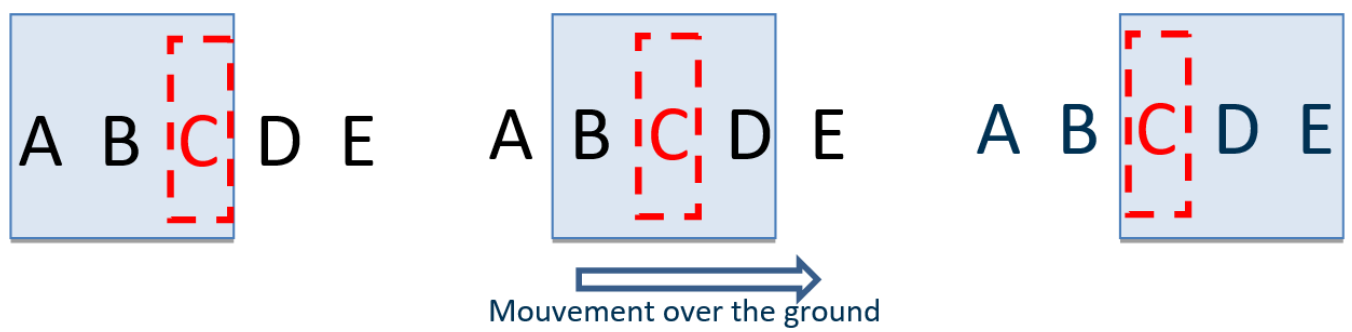

Figure 3-8: Illustration of how to take 3 images of the same ground scene. The red dashed rectangle represents the selected image area used to measure aberrations (this may include a guard band to remove telescope wobble). The letters represent the ground scene and the blue square the detector.

Focal plane sharpening works by taking a number of images (typically 3 or 4), each with different amounts of a given aberration (e.g. tilt of one of the segments). Because of the fast moving ground scene, taking identical images with different amount of aberrations may be challenging with limited satellite agility. Figure 3-8 gives an illustration of the strategy employed to take multiple identical images of a moving ground scene where only a sub-section of the pixel array is selected. The level of aberrations introduced can be controlled by the approach description in section 3.6. For each of these individual images, a sharpness metric $F$ is calculated, typical using image intensities (e.g. $F_{s q}=\sum_{x, y} I^{2}(x, y)$, where $\mathrm{I}(\mathrm{x}, \mathrm{y})$ is the image intensities). Using these data points, a curve is fitted (typically a Gaussian or quadratic fit), and the peak estimated. The peak corresponds to an estimation of the amount of aberrations present in the system. 

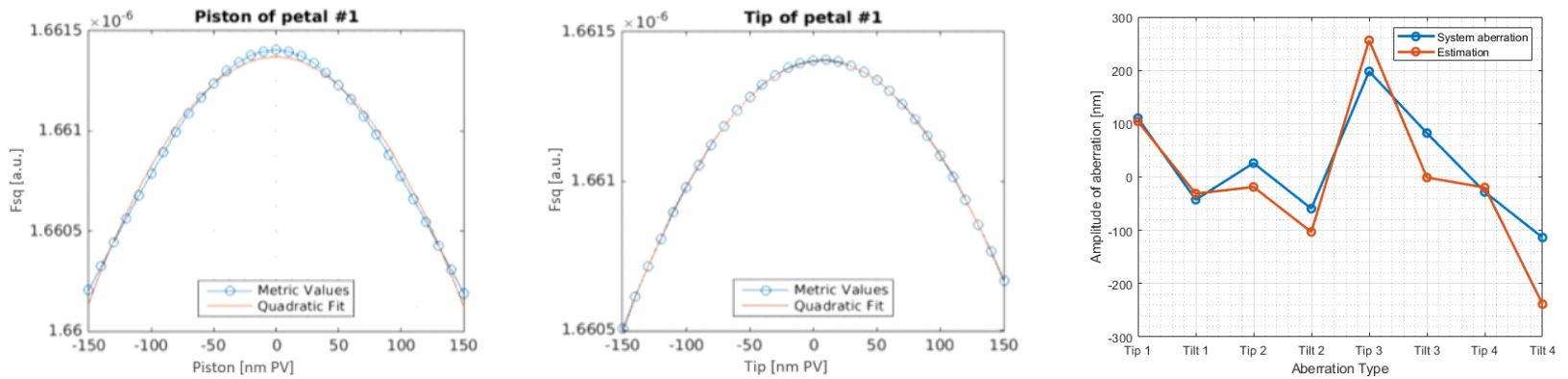

Figure 3-9: (Left and Centre) Metric versus aberrations for small aberrations. All other petals are perfectly aligned apart from the one being investigate. (Right) Illustration of simulation results using focal plane sharpening to estimate tip-tilt of all 4 segments simultaneously (all segments have piston and tip-tilt aberrations).

Figure 3-9 gives an illustration of the change in metric value with changing aberration levels (simulations); in practice typically only 3-4 data points per mode are collected to reduce time. It also illustrates the estimation of tip and tilt using focal plane sharpening. In this particular example modes where only corrected at the end of the process. This is known to produce poor results in the presence of large aberrations (such as illustrated here). Recent simulations [9] have shown that these results can be greatly improved by using a slightly different optimization scheme (i.e. a $3 \mathrm{~N}$ optimization scheme where each mode is measure and subsequently corrected sequentially, instead of a $2 \mathrm{~N}+1$ optimization scheme where modes are only corrected at the end of the process). In addition, using a Gaussian fitting function instead of a quadratic will significantly improve results. Finally, running the algorithm multiple times also improve the results, as each step benefits from the previous correction.

\section{DEPLOYABLE SECONDARY MIRROR}

\subsection{Review of concepts}

In addition to deploying the primary mirror (M1) to increase the photometric power, SNR, and angular resolution, there is a need to package and deploy the secondary mirror (M2) to minimize volume during launch. Deployment mechanisms can be used to extend the M1 to M2 separation, which increases the focal length. As we have seen section 3.1, increasing M1M2 distance can have a dramatic effect on misalignment sensitivity. Nearly every design reviewed in this study utilises a reflective optical system and typically a variant of a Cassegrain layout (see for example $[10,11])$. This design offers a large aperture and focal length by folding the light path within the CubeSat volume.

From a conceptual point of view, the different deployment concepts can be grouped broadly into four categories: Telescopic boom, Tape spring, Truss mast, and Articulated arm (see Figure 4-1 for illustration). The telescopic boom concepts commonly deploy M2 by extending an arm or tube made up of concentric and sequentially smaller components, much like a radio aerial. The deployment arms of a tape spring concept consist of an individual coiled tape that when deployed takes on a curved dimension to hold its deployed shape, much like a conventional tape measure. Truss mast concepts typically consist of multiple smaller arms and struts that build up to form a rigid lattice structure. Some of the truss mast arms can pivot such that the design can be folded into a smaller stowed volume. Articulated arm concepts typically consist of a few rigid arms that join at hinges. In the deployed state these arms are folded into a small volume, but can then fold out to the maximum end of the hinge travel in order to extend the deployment mechanism.

Key metrics by which to judge the different deployment concepts are: the deployment accuracy and repeatability, the rigidity in the deployed state and the potential to actuate the position and tip/tilt of M2 for alignment. Telescopic boom and truss mast concepts have potential to have relatively high rigidity, by the fact that they can be quite solid components. Furthermore, they offer significant design freedom in terms of baffling, light-weighting and vibration resonance dampening, by the fact that the deployment arms can be made of numerous materials, shapes and sizes. However, common challenges for these designs are relatively large stowed volumes and integrating M2 actuation mechanisms. Tape spring concepts can be designed to actuate a deployed M2 in three positional degrees of freedom as well as tip/tilt, by the fact that each tape spring leg could be actuated in length, in turn making the system behave like a hexapod. The stowed state can potentially be relatively small as well. However, it is not clear that state of the art tape spring concepts are rigid enough when deployed to maintain good image performance on board a CubeSat. Articulated arm concepts are a good solution for large scale satellites e.g. JWST, as they can deploy M2 to a fixed position with high rigidity once the arms are locked in 
place. JWST then aligns the telescope by actuating M1 segments, which on smaller CubeSat-sized satellites is a less feasible option. Research into articulated arm deployment mechanisms for CubeSat scale missions is relatively minimal.
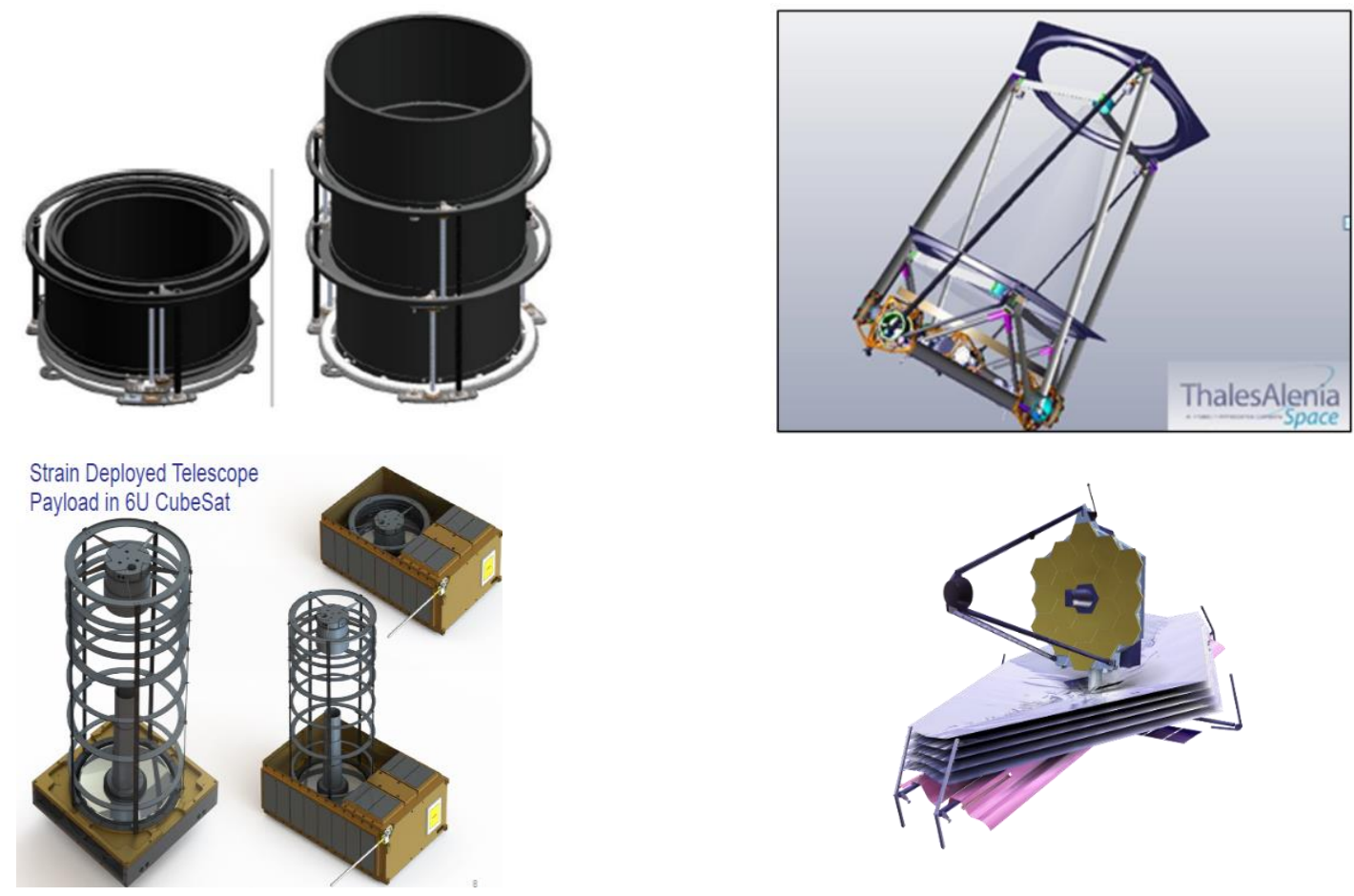

Figure 4-1: Examples of various concept for deploying M2 mirrors. From top to bottom and left to right: a telescopic boom concept from SSTL (left-stowed and right-deployed) [10]; a tape spring concept from Thales Alenia Space [12]; a Truss mast concept on a $6 \mathrm{U}$ CubeSat from NASA AMES Research Centre [13]; an articulated arm design for the JWST.

Many deployable M2 design concepts would not be applicable to nanosats (e.g. because of volume constrains). In addition, adapting them to Earth observation applications may be non-trivial due to the need of a deployable baffle. One of the main remaining issue however, is the investigation of the suitability of these systems and the level of fine actuation (i.e. with resolutions far below the micron) required to achieve diffraction-limited performance.

\subsection{Optical sensitivity for deployable CubeSats}

The analysis of the optical design presented in section 3.1 showed that the tolerances were very tight, in particular concerning the position of the secondary mirror M2. One way to reduce the number of degrees of freedom required for alignment would be to use a spherical M1 or M2. For a sphere, a decentre becomes equivalent to a tilt and can be compensated by a single degree of freedom. This appears appealing on the surface, but proves difficult to design well due to the presence of large amounts of coma aberration, requiring complex field correcting optics. Using a spherical M1 or M2 does not seem a viable solution at this stage, at least for nanosats. After modelling and analysis, we set the M2 optical diameter to $50 \mathrm{~mm}$ to avoid vignetting and excessive mass. Both M1 and M2 have non-spherical surfaces.

To reach diffraction-limited performance in the visible $(500 \mathrm{~nm})$, the overall aberration level needs to be below $\leq 36 \mathrm{~nm}$ (defining diffraction-limited system for $\lambda / 14$ ). We then set the requirement for each of the independent individual aberration contributors to $\leq 12 \mathrm{~nm}$ [14]. Finally, in order to analyse the sensitivity of M2 to misalignments, we investigate the impact of Defocus (i.e. a movement along the z-axis), Decentre (i.e. a movement along the X-Y plane), and Tilt (a rotation around the $\mathrm{X}$ or $\mathrm{Y}$ axis) on aberration levels. Because of symmetry, a rotation around the z-axis has not impact on image quality.

Figure 4-2 and Figure 4-3 show the relative changes in RMS wavefront error (WFE), which is subtracted from the RMS WFE of the nominal model. We have seen that adding a simple field corrector (e.g. designed with only a couple lenses) has the ability to reduce the nominal system RMS WFE to negligible amounts. Figure 4-2 shows the impact of defocus on final optical aberrations for 3 different M1-M2 distances (300, 400, and $500 \mathrm{~mm}$ ), assuming the use of field corrector 
capable of bringing the nominal system RMS WFE down sufficiently. Unsurprisingly, increasing the M1-M2 separation helps reducing the sensitivity to defocus (i.e. we can tolerate larger defocus values). The 3 horizontal lines, represent respectively the diffraction-limit (i.e. $36 \mathrm{~nm})$, the requirement $(12 \mathrm{~nm})$, and a goal $(9 \mathrm{~nm})$. For a M1-M2 separation of $300 \mathrm{~mm}$, a defocus of $\leq 0.7 \mu \mathrm{m}$ would still enable diffraction-limited imaging. This appears below the achievable precision of a single-use deployment system (as for example presented see Figure 3-4), and would in practice require a fine movement actuator or stage to reach this value.

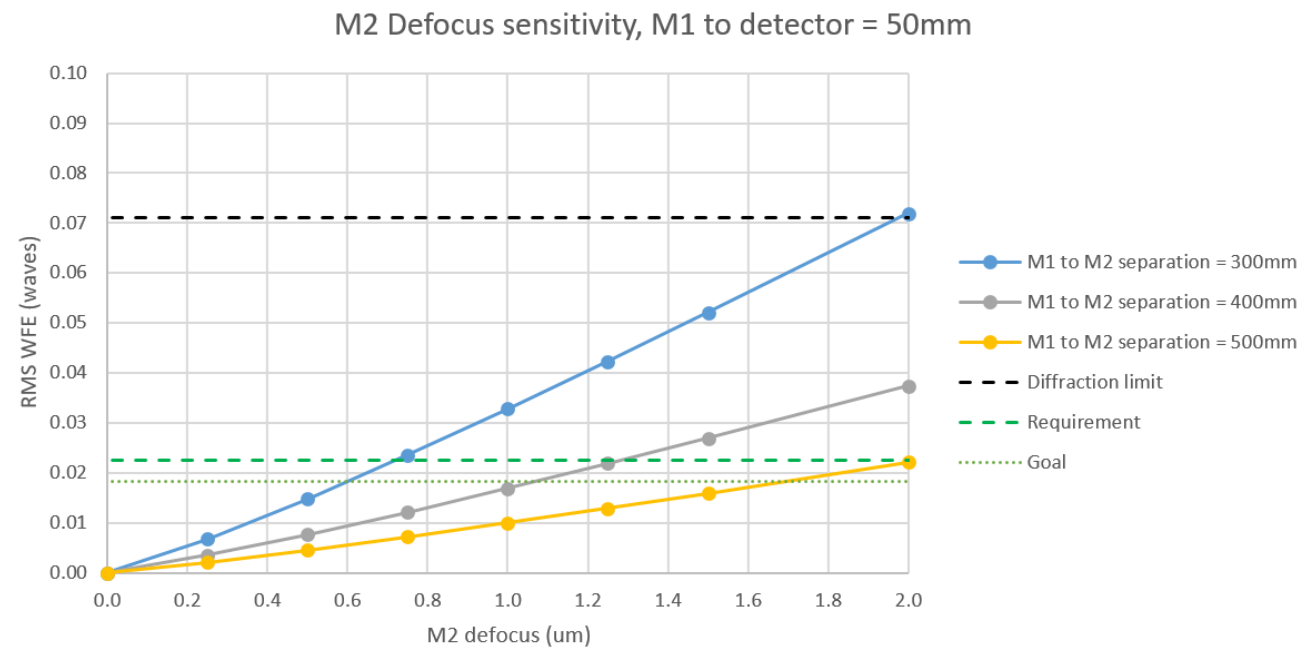

Figure 4-2: Impact of defocus on final optical aberrations for 3 different M1-M2 distances.

Figure 4-3 shows the impact of tilt on final optical aberrations for 3 different M1-M2 distances (300, 400, and $500 \mathrm{~mm}$ ), assuming the use of field corrector capable of bringing down the nominal system RMS WFE sufficiently. Again, increasing the M1-M2 separation helps in reducing the sensitivity to tilt error. For a M1-M2 separation of $300 \mathrm{~mm}$, a M2 tilt of $\leq 28 \mathrm{mrad}$ would still enable diffraction-limited imaging. This is equivalent to a $14 \mu \mathrm{m}$ PV tilt across a $50 \mathrm{~mm}$ diameter mirror, which appears within reach of a single-use deployment mechanism such as presented section 3.4.

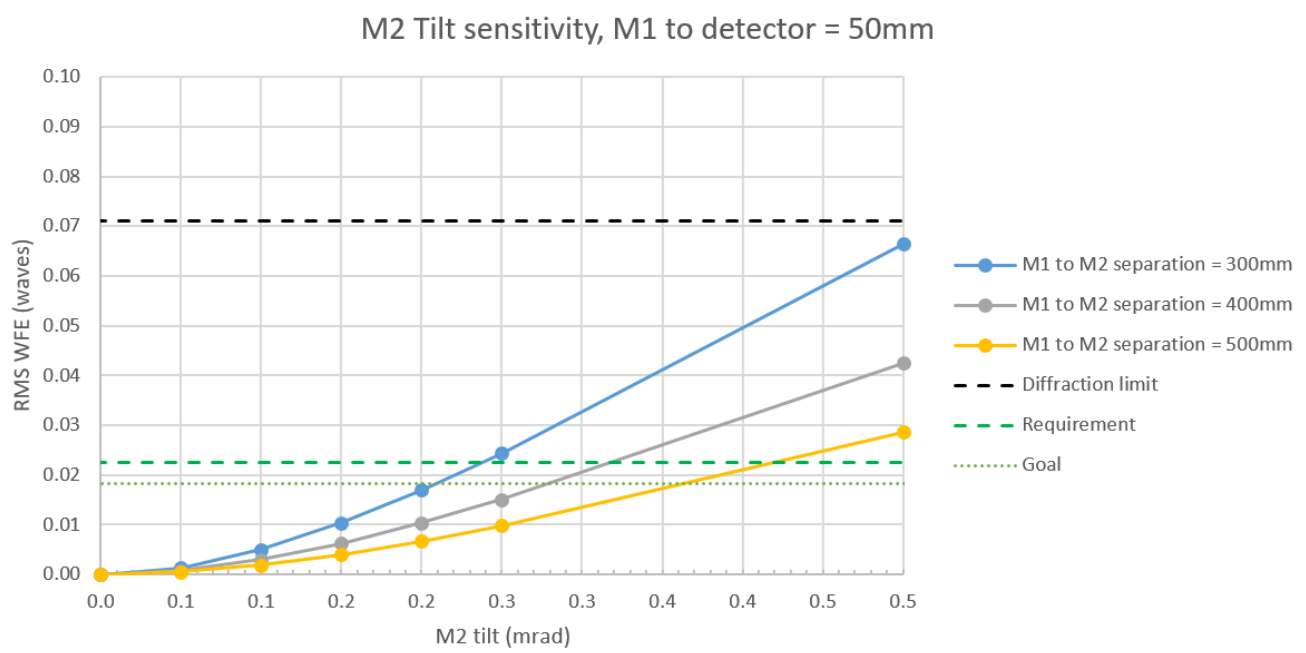

Figure 4-3: Impact of tilt on final optical aberrations for 3 different M1-M2 distances.

A similar analysis was performed on M2 decentre (data not shown). This again demonstrates the need for large M1-M2 distance to reduce sensitivity, and shows that a decentre below $\leq 10 \mu \mathrm{m}$ would still enable the system to reach the diffraction-limit. It is possible to slightly relax the tolerances by having actuation control in decenter or tip/tilt. Suitable preliminary designs to meet these requirements are currently being investigated at the UKATC. 


\section{CONCLUSION}

In this paper we presented a concept for a high-resolution Earth Observation CubeSat using a deployable primary mirrors. A laboratory opto-mechanical prototype of this concept was developed and discussed in this paper. We demonstrated the feasibility of having a segmented primary mirror that can be deployed; its position measured and manipulated with the level of accuracy required for co-phasing. The primary mirror prototype was packaged within a $1.5 \mathrm{U}$ CubeSat volume. We validated that we can deploy the mirror segments with a repeatability of less than $\pm 4.5 \mu \mathrm{m}$ (typically less than $\pm 3 \mu \mathrm{m}$ ). We demonstrated that the position of the mirrors, using the capacitive sensors and the linear piezo actuators, can be adjusted to within $\leq 10 \mathrm{~nm}$ of their optimal position; an accuracy sufficient to achieve diffraction-limited performance. Finally, we presented a sensitivity analysis of M2 misalignments and demonstrated that they are well within reach of current technology developed for phasing M1 segments.

The severe volume constrains - limited to $1.5 \mathrm{U}$ in our prototype version for fitting $\mathrm{M} 1$ - leads us to now consider a larger $4 \mathrm{U}$ alternative for the entire payload, which opens up new avenues in terms of opto-mechanical packaging, deployment strategies, and active optics correction. Using this larger volume, we now need to develop the concept further to reduce complexity (e.g. the number of active components, in the current design 12 degrees of freedom to control 4 M1 mirror segments), and take into account the volume for additional deployable M2 and baffle subsystems (we have found this unfeasible in a $2 \mathrm{U}$ payload, i.e. $1.5 \mathrm{U}$ for $\mathrm{M} 1$ and $0.5 \mathrm{U}$ for $\mathrm{M} 2$ and baffle). Performance was also limited by the actuators used in the demonstrator. Space qualified actuators are available commercially (e.g. Credat PPA40M), but coupling them to optical surfaces in a precise, reliable, and repeatable manner is challenging. Conceptual design of M2 and baffle needs to be furthered. Particular design emphasis will be placed on the deployment mechanisms and their accuracies, the resolution of the actuator mechanisms (required reach optimal optical quality), and future flight compatibility.

\section{REFERENCES}

[1] Schwartz, N., et al., "A segmented deployable primary mirror for earth observation from a CubeSat platform," in Proc. AIAA/USU Conf. Small Satellite, vol. SSC16, p. SSC16-WK-23 (2016).

[2] Schwartz, N., et al., "Laboratory demonstration of an active optics system for high-resolution deployable CubeSat," in 4S Symposium, vol. 1, pp. 1-15 (2018).

[3] Villalba, V., Kuiper, H., Eberhard Gill, "Review on thermal and mechanical challenges in the development of deployable space optics,” J. Astron. Telesc. Instrum. Syst. 6(1), 010902 (2020).

[4] Wells, M., et al. "The Mid-Infrared Instrument for the James Webb Space Telescope, VI: The Medium Resolution Spectrometer." Publications of the Astronomical Society of the Pacific, vol. 127, no. 953, 2015, pp. 646-664.

[5] Schwartz, N., Sauvage, J.-F., et al., "Sensing and control of segmented mirrors with a pyramid wavefront sensor in the presence of spiders," in Proc. AO4ELT5 (2017).

[6] Schwartz, N., Sauvage, J.-F., et al., "Analysis and mitigation of pupil discontinuities on adaptive optics performance," Proc. SPIE10703, 1070322 (2018).

[7] Patent "A moveable joint" (granted 10 June 2020).

[8] Razorbill Instruments <https://razorbillinstruments.com/zed-cap-a-compact-high-precision-displacementsensor/> (30 October 2020).

[9] Gore, Benjamin, et al. "Adaptive optics for multifocal plane microscopy." Proc. of workshop on adaptive optics for industry and medicine (2019).

[10] Gooding, D., et al. "A novel deployable telescope to facilitate a low-cost <1m GSD video rapid-revisit small satellite constellation." International Conference on Space Optics-ICSO 2018. Vol. 11180. International Society for Optics and Photonics, (2019).

[11] Allthorpe-Mullis, E., Brzozowski, W., et al. "Cubesat camera: A low cost imaging system for CubeSat platforms.” Proceedings of iCubeSat (2018). 
[12] Blanchard, L. “A tape-spring hexapod for deployable telescopes: Dynamics.” ESA Special Publication. Vol. 621. (2006).

[13]Elwood, A., Kimberly Ennico-Smith, K., and Rademacher, A., "Collapsible space telescope (CST) for nanosatellite imaging and observation." (2013).

[14] Sauvage, J.-F., et al. "AZIMOV: First error budget of a deployable CubeSat telescope," Space Telescopes and Instrumentation 2020: Ultraviolet to Gamma Ray. Vol. 11444. International Society for Optics and Photonics, 2020.

Proc. of SPIE Vol. $114431144331-13$

Downloaded From: https://www.spiedigitallibrary.org/conference-proceedings-of-spie on 04 Jan 2021 Terms of Use: https://www.spiedigitallibrary.org/terms-of-use 